\title{
A Diffusion Model for Energy Harvesting Sensor Nodes
}

\author{
Omer H. Abdelrahman and Erol Gelenbe \\ Intelligent Systems and Networks \\ Department of Electrical \& Electronic Engineering \\ Imperial College, London SW7 2BT, UK \\ Email: \{o.abd06, e.gelenbe\}@imperial.ac.uk
}

\begin{abstract}
Energy harvesting has recently attracted much interest due to the emergence of the Internet of Things, and the increasing need to operate wireless sensing devices in challenging environments without much human intervention and maintenance. This paper presents a novel approach for modeling the performance of an energy harvesting wireless sensor node, which takes into account fluctuations in the amount of energy extracted from the environment, energy loss due to battery leakage, as well as the energy cost of sensing, data processing and communication. The proposed approach departs from the common queueing-theoretic framework used in the literature, and instead uses Brownian motion to represent more accurately the time evolution of the distribution of the node's battery level. The paper derives some performance measures of interest along with the stationary solution of the system, and discusses possible directions for reducing the number of parameters and states of the model without compromising accuracy.
\end{abstract}

Keywords-Energy harvesting; Internet of Things; wireless sensor networks; diffusion process; Fokker-Planck equation

\section{INTRODUCTION}

There are a wide range of technologies that are being developed which rely on the availability of efficient energy harvesting systems and algorithms [1], [2]. Examples include structural and equipment monitoring, medical implants, wearable electronics, and asset tracking to name just a few. Ideally, such sensing devices need to operate in an energy neutral manner [3], by adapting data gathering and transmission processes to the availability of renewable and intermittent sources of energy.

In this paper, we study the time evolution of the energy stored in a wireless sensor node that harvests energy and uses the energy to collect and forward data. The arrival of energy and of data packets to the node are both assumed to be random processes: energy is harvested from random sources such as light, motion, and temperature, and data accumulates into the node, also at random, through sensing or reception from other nodes. However, while data is measured in terms of discrete packets, energy is quantified or measured in continuous units. Our aim is to provide a fine-grained mathematical framework for optimizing the performance of these systems, which complements recent coarse-grained approaches based on queueing theory [4]-
[8], or based on fluid flow approximations [9], [10] that may not capture adequately the stochastic nature of energy availability.

Much work has been devoted in recent years to the fundamental analysis and optimization methods that can be used in energy harvesting wireless communications. A common approach in the literature is to represent the energy stored by a node in discrete units, which are called energy packets in [6]-[8], [11] where an energy packet is defined as the minimum amount of energy needed to transmit a single data packet. Intuitively, one would expect that the energy flow into the node should somehow balance the flow of data packets. However, it has been shown in [6] that if the flows of energy and of data packets are exactly balanced, then the system exhibits an unstable behavior, such that the variance of the imbalance between data and energy packets increases indefinitely with time. This is a frequently encountered problem in queueing theory [12] concerning the stability of the synchronization of two independent streams, when the synchronization time is negligibly small. Smart scheduling policies have been suggested [13] to adapt sensing and transmission power to the energy and channel conditions, thus providing stability and good performance.

In another related work [14], the state of battery for a renewable-operated wireless access point is modeled as a G/G/1 queue with arbitrary arrivals and departures of unit energy. Diffusion approximation is then used to analyze the time-dependent behavior of the buffer. Here we go further by incorporating the interactions between the energy and data buffers, as well as temporal variations, into a novel diffusion model while providing a simpler analytical framework than previous attempts [15], [16] to address these issues.

The rest of the paper is organized as follows. Section II introduces the mathematical model along with performance measures of interest, and derives the stationary solution of the system under specific boundary conditions. Numerical examples based on the steady-state analysis are presented in Section III. Finally, the paper concludes in Section IV with a discussion of possible directions for reducing the complexity of the model so as to obtain more explicit analytical results. 


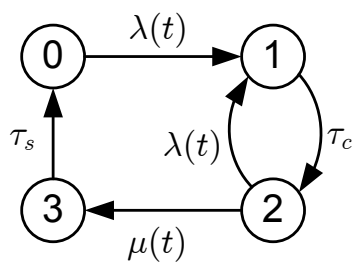

Figure 1. The four states and associated transition rates in the model. Within each state, battery level varies according to a distinct diffusion process.

\section{Mathematical Model}

Consider a wireless harvesting sensor node with energy storage of size $B>0$, and let $0 \leq X_{t} \leq B$ be the amount of energy stored at time $t$. We denote by $Y_{t} \in\{0,1,2,3\}$ the state of the node at $t$ where:

$$
Y_{t}= \begin{cases}0, & \text { if node is idle with no data buffered } \\ 1, & \text { if node is collecting data, } \\ 2, & \text { if node is idle with data buffered } \\ 3, & \text { if node is transmitting data, }\end{cases}
$$

Energy harvesting is assumed to be active in all of the four states. We model $\left\{X_{t}, Y_{t}: t \geq 0\right\}$ as a diffusion process [17]-[21] in which the mean change in the amount of energy stored in a small time interval $[t, t+\Delta t)$ is $b_{i}(t) \Delta t$, while the variance of the energy over the same time interval is $c_{i}(t) \Delta t$ :

$$
\begin{aligned}
b_{i}(t) & =\lim _{\Delta t \rightarrow 0} \frac{E\left[X_{t+\Delta t}-X_{t} \mid Y_{t}=i\right]}{\Delta t}, \\
c_{i}(t) & =\lim _{\Delta t \rightarrow 0} \frac{E\left[\left(X_{t+\Delta t}-X_{t}\right)^{2}-E\left[X_{t+\Delta t}-X_{t}\right]^{2} \mid Y_{t}=i\right]}{\Delta t}
\end{aligned}
$$

A schematic representation of the model is presented in Fig. 1, illustrating the transition rates between the four states: data is accumulated at rate $\lambda(t)$, whenever the node is idle, and each sensing process takes on average $\tau_{c}^{-1}$; the data buffer is emptied with rate $\mu(t)$ and the average transmission time is $\tau_{s}^{-1}$.

Fig. 2 shows a simulation of the evolution of the energy stored in a solar harvesting sensor node, where data is gathered and transmitted at frequency that follows the diurnal cycle of light intensity. Our model aims to characterize the distribution of such energy profile.

When $Y_{t}=0$, the sensor node is idle, does not have any data buffered and is harvesting energy. Accordingly, the time-dependent parameters $b_{0}(t)$ and $c_{0}(t)$ capture the following energy aspects of the node's operation:

- Energy extracted from the environment, which typically varies with time.

- Leakage from the energy storage.

- Baseline power consumption of the node, excluding data processing and communication.

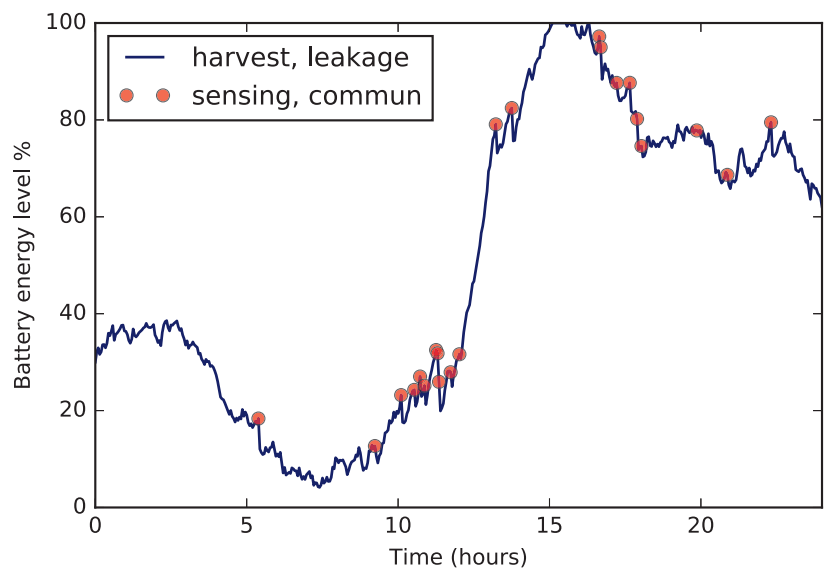

Figure 2. A simulation of the energy level of a solar harvesting node during a 24-hour period. The red circles indicate drops in energy due to sensing or communication activities which happen more frequently as the intensity of light (peaking at noon) increases.

Thus, the drift $b_{0}(t)$ can be sometimes positive when the amount of energy being harvested exceeds on average the node's idle consumption, while in other times it can be negative, e.g. during the night in the case of solar harvesting.

The node will leave the idle state 0 at time $t$ with rate $\lambda(t)$ and move to state 1 , where it will collect data by either i) receiving a transmission from a neighboring node, or ii) sampling its own sensor and processing the sampled value. This data collection process is assumed to last for an exponentially distributed time of average $\tau_{c}^{-1}$, during which the energy level varies according to a diffusion process with parameters $b_{1}(t)$ and $c_{1}(t)$, which is the result of both energy harvesting and data collection. Note, however, $\tau_{c}^{-1}$ will typically be very small while $b_{1}(t)<<0$ to represent the negative jump in the energy level due to data reception or acquisition as shown in Fig 2.

The data collected by the node is subsequently stored in a data buffer, and the state of the node changes to 2 . Since the node may have a different duty cycle when its data buffer is occupied than when it is empty, the diffusion parameters in states 2 and 0 can be different. From state 2 , the node may again move to state 1 , with rate $\lambda(t)$, in order to gather and process more samples (e.g. to improve quality of information) or it may move to state 3 with rate $\mu(t)$ signifying the beginning of a transmission. The sending of data occurs over an exponentially distributed time with mean $\tau_{s}^{-1}$ during which energy level diffuses with parameters $b_{3}(t)$ and $c_{3}(t)$, independently of the number of data collection operations performed since the previous transmission. Thus, we assume that the node may gather and process a number of samples (i.e. it visits states 1 and 2 multiple times), before finally sending all data in one packet in state 3. After that the buffer becomes empty and the node returns to state 0. Again, the negative jump in energy level 
due to a transmission can be represented by a small duration $\tau_{s}^{-1}$ and a negative drift $b_{3}(t)<<0$.

We do not make any assumptions regarding the values (and relative magnitude) of the diffusion parameters in states 1 and 3, since the energy costs of sensing and of wireless transmission vary according to the application domain, the sensing environment, the type of raw input used by the sensor and so on. For example, transmission consumes most of the energy for low-quality, low-rate sensor nodes, and for simple sensing modalities such as temperature and light. On the other hand, sensing can be the most energy demanding process for some acoustic and seismic sensing applications that require high-rate and high-resolution analog-to-digital conversion [22].

\section{A. System of Differential Equations}

Let $f_{i}(x, t)$ be the probability density that the sensor node has energy level $x$ and in occupancy state $i$ at time $t$. Mathematically, $f_{i}(x, t)$ is defined as

$$
f_{i}(x, t)=\lim _{\Delta x \rightarrow 0} \frac{\operatorname{Pr}\left[x \leq X_{t} \leq x+\Delta x, Y_{t}=i\right]}{\Delta x} .
$$

The time evolution of $f_{i}(x, t)$ is governed by the coupled diffusion or Fokker-Planck equations:

$$
\begin{aligned}
& \partial_{t} f_{0}=-\partial_{x} J_{0}-\lambda(t) f_{0}+\tau_{s} f_{3}, \\
& \partial_{t} f_{1}=-\partial_{x} J_{1}-\tau_{c} f_{1}+\lambda(t)\left[f_{0}+f_{2}\right], \\
& \partial_{t} f_{2}=-\partial_{x} J_{2}-[\lambda(t)+\mu(t)] f_{2}+\tau_{c} f_{1}, \\
& \partial_{t} f_{3}=-\partial_{x} J_{3}-\tau_{s} f_{3}+\mu(t) f_{2},
\end{aligned}
$$

where we used the shortened notation $f_{i}$ and $J_{i}$ to denote $f_{i}(x, t)$ and $J_{i}(x, t)$, respectively, with $J_{i}$ being the probability flux in the positive direction across a level $x$ at time $t$ for the $i$-th state:

$$
J_{i}(x, t)=-\left[\frac{c_{i}(t)}{2} \partial_{x} f_{i}(x, t)-b_{i}(t) f_{i}(x, t)\right] .
$$

The system of linear second-order partial differential equations (1) can be written in matrix form as:

$$
\nabla_{t} f=\mathbf{C} \nabla_{x}^{2} f-\mathbf{B} \nabla_{x} f-\mathbf{A} f
$$

with $f=\left[f_{0}(x, t), f_{1}(x, t), f_{2}(x, t), f_{3}(x, t)\right]^{T}$ and

$$
\begin{aligned}
\mathbf{A} & =\left[\begin{array}{cccc}
\lambda(t) & 0 & 0 & -\tau_{s} \\
-\lambda(t) & \tau_{c} & -\lambda(t) & 0 \\
0 & -\tau_{c} & \lambda(t)+\mu(t) & 0 \\
0 & 0 & -\mu(t) & \tau_{s}
\end{array}\right], \\
\mathbf{B} & =\operatorname{diag}\left[b_{0}(t), \cdots, b_{3}(t)\right], \mathbf{C}=\frac{1}{2} \operatorname{diag}\left[c_{0}(t), \cdots, c_{3}(t)\right] .
\end{aligned}
$$

The solution of (3) requires the introduction of appropriate initial and boundary conditions. Specifically, if the energy stored at the beginning of operation is $x_{0}$ and the data buffer is empty, then we end up with the following initial conditions:

$$
f_{i}(x, 0)= \begin{cases}\delta\left(x-x_{0}\right), & \text { for } i=0 \\ 0, & \text { otherwise }\end{cases}
$$

where $\delta\left(x-x_{0}\right)$ is the Dirac delta function at $x_{0}$. Since the energy level cannot be negative or exceed $B$, the probability current across the lower and upper boundaries of the energy storage must be zero:

$$
\lim _{x \rightarrow 0}-J_{i}(x, t)=\lim _{x \rightarrow B} J_{i}(x, t)=0, \quad \forall i
$$

We also have that the sum of the probabilities at any time $t$ must be one:

$$
\int_{0}^{B} \sum_{i=0}^{3} f_{i}(x, t) d x=1
$$

\section{B. Performance Metrics}

One performance measure of interest is the average energy level at time $t$ which can be computed as:

$$
\mathcal{E}(t)=\int_{0}^{B} x \sum_{i=0}^{3} f_{i}(x, t) d x
$$

One may also be interested in evaluating some notion of a failure rate relating to insufficient energy for sensing or communication:

$\gamma(t)=\int_{0}^{\varepsilon_{c}}\left[f_{0}(x, t)+f_{2}(x, t)\right] \lambda(t) d x+\int_{0}^{\varepsilon_{s}} f_{2}(x, t) \mu(t) d x$,

where $\varepsilon_{c}$ and $\varepsilon_{s}$ denote, respectively, the minimum energy required to collect and send data successfully.

\section{Stationary Analysis}

As a first step towards analyzing the performance of the sensor node, we consider the steady-state solution of the model when the different parameters do not vary much with $t$ over a relatively large time window $T$. This can be relevant to applications where major fluctuations in the rate of energy harvesting occur at a granularity of minutes or an hour, within which smaller fluctuations are captured adequately by a fixed variance, i.e. $b_{i}(t) \simeq b_{i}$ and $c_{i}(t) \simeq c_{i}$ for $t \in\left[t_{0}, t_{0}+T\right]$. In such instances, the node may use fixed data rates ( $\lambda$ for sensing and $\mu$ for communication) within the window of size $T$ and the time-dependent parameters of the model can be approximated by piece-wise constant functions. If $T$ is long enough the system can attain stationarity, i.e. $\lim _{t \rightarrow \infty} \nabla_{t} f=0$ and $\lim _{t \rightarrow \infty} f(x, t)=f(x)$ so that (3) becomes:

$$
f^{\prime \prime}(x)-\mathbf{C}^{-1} \mathbf{B} f^{\prime}(x)-\mathbf{C}^{-1} \mathbf{A} f(x)=0 .
$$

This linear system of 4 second-order ordinary differential equations can be transformed into a set of 8 first-order equations as follows:

$$
\frac{d}{d x}\left[\begin{array}{c}
f \\
f^{\prime}
\end{array}\right]=\left[\begin{array}{cc}
\mathbf{0} & \mathbf{I} \\
\mathbf{C}^{-1} \mathbf{A} & \mathbf{C}^{-1} \mathbf{B}
\end{array}\right]\left[\begin{array}{c}
f \\
f^{\prime}
\end{array}\right] \equiv \mathbf{M}\left[\begin{array}{l}
f \\
f^{\prime}
\end{array}\right],
$$


where $\mathbf{0}$ and $\mathbf{I}$ are the zero and identity matrices, respectively. The solution to (7) is given by:

$$
f(x)=\sum_{j}\left[\alpha_{j} \Re\left(e^{\xi_{j} x} \phi_{j}\right)+\beta_{j} \Im\left(e^{\xi_{j} x} \phi_{j}\right)\right],
$$

where $\Re, \Im$ denote the real and imaginary parts of a complex number; $\xi_{j}$ are the eigenvalues of $\mathbf{M} ; \phi_{j}=\left[\phi_{j 0}, \cdots, \phi_{j 3}\right]^{T}$ are vectors formed by taking the first four elements of the corresponding eigenvectors of $\mathbf{M}$ (as we are interested in the solution for $f$ and not $f^{\prime}$ ); and $\alpha_{j}, \beta_{j}$ are constants that can be obtained from the boundary and normalization conditions (5)-(6). Since the characteristic equation $|\mathbf{M}-\xi \mathbf{I}|=0$ has real coefficients, any complex roots must occur in conjugate pairs. Each of these pairs will give, up to sign, the same solutions and therefore only one of the two eigenvalues is required. Note also that $f(x)$ takes a slightly different form than above if there are repeated eigenvalue-vector pairs, but this special case will not be addressed here.

Let $\xi_{j}=r_{j}+s_{j} \sqrt{-1}$ be a complex eigenvalue with $\phi_{j}=$ $u_{j}+v_{j} \sqrt{-1}$, and define the vectors:

$$
\begin{aligned}
w_{j}(x) & =e^{r_{j} x}\left[u_{j} \cos \left(s_{j} x\right)-v_{j} \sin \left(s_{j} x\right)\right], \\
\sigma_{j}(x) & =e^{r_{j} x}\left[u_{j} \sin \left(s_{j} x\right)+v_{j} \cos \left(s_{j} x\right)\right],
\end{aligned}
$$

which are the real and imaginary parts of $e^{\xi_{j} x} \phi_{j}$, then the probability density $f(x)$ can be written as:

$$
f(x)=\sum_{\xi_{k} \in \mathbb{R}} \alpha_{k} e^{\xi_{k} x} \phi_{k}+\sum_{\xi_{j} \in \mathbb{C}}\left[\alpha_{j} w_{j}(x)+\beta_{j} \sigma_{j}(x)\right] .
$$

Applying the boundary conditions (5) at $x=0$ and $x=B$ for $i \in\{0,1,2,3\}$ yields:

$$
\begin{aligned}
0 & =\sum_{\xi_{k} \in \mathbb{R}} \alpha_{k}\left(\xi_{k}-\frac{2 b_{i}}{c_{i}}\right) e^{\xi_{k} x} \phi_{k i} \\
& -\sum_{\xi_{j} \in \mathbb{C}} \alpha_{j}\left[s_{j} \sigma_{j i}(x)-\left(r_{j}-\frac{2 b_{i}}{c_{i}}\right) w_{j i}(x)\right] \\
& +\sum_{\xi_{j} \in \mathbb{C}} \beta_{j}\left[s_{j} w_{j i}(x)+\left(r_{j}-\frac{2 b_{i}}{c_{i}}\right) \sigma_{j i}(x)\right], x=0, B
\end{aligned}
$$

while the normalization condition (6) gives:

$$
\begin{aligned}
1 & =\sum_{i=0}^{3}\left\{\sum_{\xi_{k} \in \mathbb{R}} \frac{\alpha_{k}}{\xi_{k}}\left(e^{\xi_{k} B}-1\right) \phi_{k i}\right. \\
& +\sum_{\xi_{j} \in \mathbb{C}} \frac{\alpha_{j}}{r_{j}^{2}+s_{j}^{2}}\left[r_{j} w_{j i}(B)+s_{j} \sigma_{j i}(B)-r_{j} u_{j i}-s_{j} v_{j i}\right] \\
& \left.+\sum_{\xi_{j} \in \mathbb{C}} \frac{\beta_{j}}{r_{j}^{2}+s_{j}^{2}}\left[r_{j} \sigma_{j i}(B)-s_{j} w_{j i}(B)-r_{j} v_{j i}+s_{j} u_{j i}\right]\right\} .
\end{aligned}
$$

The constants $\alpha_{\ell}, \beta_{\ell}$ are then obtained by solving the system of linear equations (9)-(10).

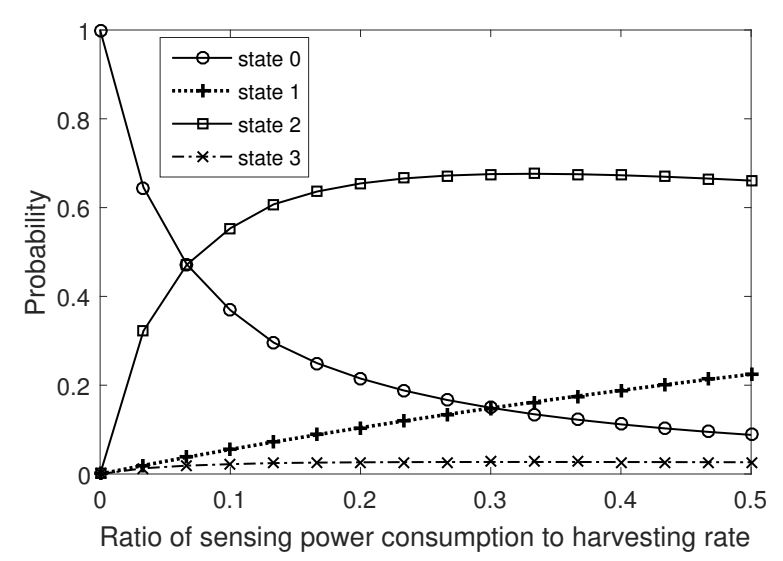

Figure 3. The probability of being in state $i$ versus the ratio of average sensing power consumption $\lambda\left|b_{1}\right| \tau_{c}^{-1}$ to average energy harvesting rate $b_{0}$.

\section{NumeriCAL RESUlts}

In Fig. 3, we plot the probability of being in state $i$ as a function of $\lambda\left|b_{1}\right| \tau_{c}^{-1} / b_{0}$ - the ratio of average power consumption due to sensing to average harvesting rate - using the following parameters: $\left(b_{2}, c_{2}\right)=\left(b_{0}, c_{0}\right)=(0.03,0.01)$; $\left(b_{3}, c_{3}\right)=\left(b_{1}, c_{1}\right)=(-0.05,0.02) ; \tau_{c}=\tau_{s}=5 ; B=1$; $\mu=0.2$; and $\lambda \in[0,1.6]$. The numerator of the horizontal axis is computed as the product of the data gathering rate $\lambda$ and the average energy expended per sensing operation $\left|b_{1}\right| \tau_{c}^{-1}$, which provides an upper-bound by assuming sensing is active in all of the four states; the denominator $b_{0}$ represents the average energy harvesting rate if we neglect leakage and standby power consumption. As $\lambda$ is increased, we see that the node spends more time acquiring data (states 1 and 2) and less idle time in 0 while the data throughout, which is proportional to the fraction of time in state 3 , remains largely unaffected because the node does not transmit individual packets but rather combines them into fewer transmissions. However, although a high sensing rate can improve quality of information, it reduces the amount of energy available to the node for sensing and transmission (as shown in Fig. 4), thus presenting an interesting trade-off which requires further exploration.

\section{FUTURE WORK}

This paper presented a pure diffusion model for batteryoperated wireless sensor nodes that are charged by renewable energy sources such as wind or solar. The diffusion-based formulation allowed us to characterize the time evolution of the energy stored in the battery using four coupled diffusion equations which can be solved with standard analyticalnumerical methods. In future work, we will investigate variations of the model that capture the same dynamics of the node's operation but with fewer parameters and states, which may allow the eigen-decomposition step of the solution to be performed analytically. One possible direction involves 


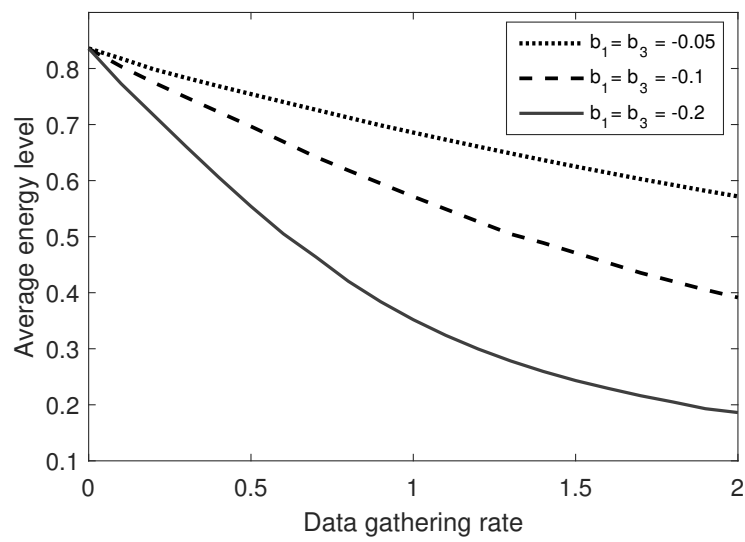

Figure 4. Average energy stored in the battery versus data gathering rate $\lambda$, using the same parameters as before but different sensing $b_{1}$ and transmission $b_{3}$ power consumption costs.

combining discrete Poisson jumps, to represent the sudden drops in energy level due to sensing or communication, with background stochastic diffusion for the energy harvesting and leakage processes. Such jump-diffusion models are used extensively in other application domains [23] such as financial engineering, mathematical biology and medicine, but the energy harvesting problem presents unique challenges due to the time-dependence of parameters and the presence of boundary conditions that may render closed-form solutions elusive.

\section{REFERENCES}

[1] E. Gelenbe, D. Gesbert, D. Gunduz, H. Kulah, and E. UysalBiyikoglu, "Energy harvesting communication networks: Optimization and demonstration (the e-crops project)," in Proc. TIWDC'13, Genoa, Italy, Sep. 2013, pp. 1-6.

[2] J. Gubbi, R. Buyya, S. Marusic, and M. Palaniswami, "Internet of things (IoT): A vision, architectural elements, and future directions," Future Gener. Comp. Sy., vol. 29, no. 7, pp. 1645-1660, 2013.

[3] A. Kansal, J. Hsu, S. Zahedi, and M. B. Srivastava, "Power management in energy harvesting sensor networks," $A C M$ Trans. Embed. Comput. Syst., vol. 6, no. 4, Sep. 2007.

[4] A. Seyedi and B. Sikdar, "Modeling and analysis of energy harvesting nodes in wireless sensor networks," in Proc. Allerton'08, Urbana-Champaign, IL, Sep. 2008, pp. 67-71.

[5] A. Tandon and M. Motani, "Has green energy arrived? delay analysis for energy harvesting communication systems," in Proc. SECON'14, Singapore, Jun. 2014, pp. 582-590.

[6] E. Gelenbe, "Synchronising energy harvesting and data packets in a wireless sensor," Energies, vol. 8, no. 1, pp. 356-369, 2015.

[7] E. Gelenbe and A. Marin, "Interconnected wireless sensors with energy harvesting," in Proc. ASMTA'15, ser. LNCS, vol. 9081. Albena, Bulgaria: Springer, May 2015, pp. 87-99.
[8] E. Gelenbe and Y. M. Kadioglu, "Energy loss through standby and leakage in energy harvesting wireless sensors," in Proc. CAMAD'15, Guildford, UK, Sep. 2015, pp. 231-236.

[9] N. Gautam and A. Mohapatra, "Efficiently operating wireless nodes powered by renewable energy sources," IEEE J. Sel. Areas Commun., vol. 33, no. 8, pp. 1706-1716, Aug. 2015.

[10] O. Galinina, K. Mikhaylov, S. Andreev, A. Turlikov, and Y. Koucheryavy, "Smart home gateway system over bluetooth low energy with wireless energy transfer capability," EURASIP J. Wirel. Comm., vol. 2015, no. 1, pp. 1-18, 2015.

[11] E. Gelenbe and E. T. Ceran, "Energy packet networks with energy harvesting," IEEE Access, vol. 4, pp. 1321-1331, 2016.

[12] D. G. Kendall, "Some problems in the theory of queues," J. R. Stat. Soc. Series B Stat. Methodol., vol. 13, no. 2, pp. 151-185, 1951.

[13] V. Sharma, U. Mukherji, V. Joseph, and S. Gupta, "Optimal energy management policies for energy harvesting sensor nodes," IEEE Trans. Wireless Commun., vol. 9, no. 4, pp. 1326-1336, Apr. 2010

[14] L. X. Cai, H. V. Poor, Y. Liu, T. H. Luan, X. Shen, and J. W. Mark, "Dimensioning network deployment and resource management in green mesh networks," IEEE Wireless Commun., vol. 18, no. 5, pp. 58-65, Oct. 2011.

[15] M. Y. Naderi, S. Basagni, and K. R. Chowdhury, "Modeling the residual energy and lifetime of energy harvesting sensor nodes," in Proc. IEEE GLOBECOM'12, Anaheim, CA, Dec. 2012, pp. 3394-3400.

[16] J. M. Jornet and I. F. Akyildiz, "Joint energy harvesting and communication analysis for perpetual wireless nanosensor networks in the terahertz band," IEEE Trans. Nanotechnol., vol. 11, no. 3, pp. 570-580, May 2012.

[17] E. Gelenbe, "On approximate computer system models," $J$. ACM, vol. 22, pp. 261-269, Apr. 1975.

[18] - "Search in unknown random environments," Phys. Rev. $E$, vol. 82, no. 6, p. 061112, Dec. 2010.

[19] O. H. Abdelrahman and E. Gelenbe, "Packet delay and energy consumption in non-homogeneous networks," Comput. J., vol. 55, no. 8, pp. 950-964, Aug. 2012.

[20] - "Time and energy in team-based search," Phys. Rev. E vol. 87, no. 3, p. 032125, Mar. 2013.

[21] E. Gelenbe and O. H. Abdelrahman, "Search in the universe of big networks and data," IEEE Netw., vol. 28, no. 4, pp. 20-25, Jul. 2014.

[22] V. Raghunathan, S. Ganeriwal, and M. Srivastava, "Emerging techniques for long lived wireless sensor networks," IEEE Commun. Mag., vol. 44, no. 4, pp. 108-114, Apr. 2006.

[23] F. B. Hanson, Applied stochastic processes and control for jump-diffusions: modeling, analysis, and computation, ser. Advances in design and control. SIAM, Nov. 2007, vol. 13. 